\title{
Constrained Capacity of Delay-Limited Wireless Transceivers
}

\author{
Jos Akhtman, Robert Maunder and Lajos Hanzo \\ School of ECS., Univ. of Southampton, SO17 1BJ, UK. \\ Email: $\{$ ya,rm,lh\}@ecs.soton.ac.uk, http://www-mobile.ecs.soton.ac.uk
}

\begin{abstract}
In this paper we devise an analytical framework for the characterization of wireless transceivers. Subsequently, we utilize the proposed methodology in order to calculate the constrained capacity of the delay-limited wireless transceivers. We demonstrate that the realistic constrained capacity may be significantly lower than the idealized unconstrained Shannon capacity. Furthermore, the constrained capacity of a delay-limited transceiver may vary significantly depending on the particular combination of application scenario and channel conditions.
\end{abstract}

\section{INTRODUCTION}

Following the recent advances in our understanding of wireless communications networks, it becomes increasingly evident that no single transmission scheme or signalling strategy may be identified as optimum over the entire range of possible system scenarios and channel conditions. Instead, many authors suggest [1], [2] that a class of locally-optimum transceiver schemes may be necessary in order to achieve a range of desirable performance points in a communication medium statistically described by an ensemble of system and service scenarios. Pursuing this approach becomes increasingly feasible in the wake of emerging technological concepts, including multimode terminals [3], software-defined radios (SDR) and cognitive radios (CR) [4].

Against this background, it seems apparent that a unified methodology is necessary, which would facilitate a seamless characterization of a wide range of wireless transceiver schemes over the entire domain of communications media, service scenarios and channel conditions, which should be considered in the context of multifunctional heterogeneous wireless networks. Correspondingly, in this paper we propose an analytical framework, for the development of such a methodology. The proposed framework is then utilized in order to derive a method of calculating the constrained capacity of delay-limited wireless transceivers. We demonstrate that the capacity, constrained by a set of realistic system and channel characteristics, may be significantly lower than the idealized unconstrained Shannon capacity. Furthermore, the constrained capacity of a delay-limited transceiver may vary significantly depending on the particular combination of the application scenario and channel conditions. It should be noted that this manuscript constitutes a proof of concept and relies on a number of important conjectures, which require a more detailed theoretical, as well as empirical justification. The thorough analysis of these propositions will form part of our future work.

\section{Characterization of Generic Transceivers}

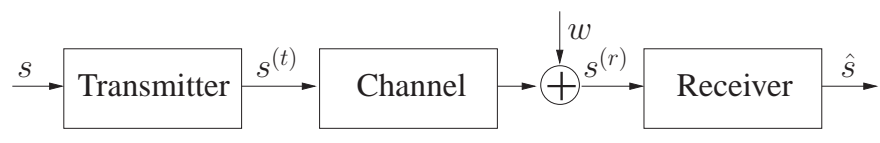

Fig. 1. Generic transceiver.
Let us consider the generic digital communication system depicted in Figure 1 At the input of our generic transceiver we have an information source, which generates a stream of information bits $s[n]$. For simplicity, in this paper we will limit ourselves to considering an equiprobable discrete binary source, although our results may be readily extended to more generic scenarios.

The entire transmission-reception process of Figure 1 may be thus interpreted as a mapping $\mathcal{S}: \mathbb{F}_{2}^{k} \rightarrow \mathbb{F}_{2}^{k}$ between the binary input information message $s$ and the output a posteriori estimated message $\hat{s}$. Furthermore, since the generic transceiver of Figure 1 is constituted by the transmitter, the channel as well as the receiver, the mapping $\mathcal{S}$ may be expressed as a serial concatenation of the corresponding mapping operations, yielding

$$
\hat{\boldsymbol{s}}=\mathcal{S}(s)=\mathcal{S}^{(r)}\left[\boldsymbol{H} \mathcal{S}^{(t)}(s)+\boldsymbol{w}\right],
$$

where the deterministic, but generally non-linear operators $\mathcal{S}^{(t)}$ and $\mathcal{S}^{(r)}$ denote the transmission and reception operations respectively, while the non-deterministic linear channel operator $\boldsymbol{H}$ as well as the additive component $\boldsymbol{w}$ represent the channel-induced signal transformation and the white Gaussian noise.

1) Channel Characteristics: Consider a hypothetic physical environment, which may be characterized by the set $\nu=$ $\{x, y, \cdots, z\}$ of physical quantities describing different aspects of its behaviour. A simplified description of the transmission medium is typically referred to as the channel model. A well-defined channel model may be readily translated into an unambiguous description of both the structure and the statistical properties of the corresponding linear operator $\boldsymbol{H}$ of Equation (1). For example, the pair of quantities $\nu=\left\{f_{D}, \tau_{m}\right\}$ corresponds to a simple model describing a family of time-variant multipath channels, which are typically characterized by the maximum Doppler spread of $f_{D}$ and the maximum delay spread of $\tau_{m}$.

2) Application Characteristics: Subsequently, let us consider a typical usage scenario, such as text messaging, voice communications, or WWW browsing. Each of the above services may be characterized by a set of QoS requirements, including for instance, the minimum data rate $R$, the maximum latency $T$, the maximum tolerable probability of error $P_{e}$, etc. Additionally, in many practical cases, the application scenario is also constrained by the maximum channel bandwidth $B$ available for communications, as well as the maximum computational processing power $\Omega$, which may be handled by the communicating terminals. In the case of multipleinput multiple-output (MIMO) systems, the application scenario may include the spacial dimensions, constituted by the numbers $n_{t}$ and $n_{r}$ of transmit and receive antennas. Correspondingly, let us define the set of parameters $\kappa=\left\{T, B, R, P_{e}, \Omega, n_{t}, n_{r}\right\}$ as the application scenario.

3) Transceiver Characteristics: Let us now define a transceiver scheme $\mathcal{S}$ as a pair of legitimate deterministic mappings $\left\{\mathcal{S}^{(t)}, \mathcal{S}^{(r)}\right\}$ of Equation (1), which unambiguously describe the

${ }^{1}$ Here, we assume a simple multipath channel model characterized by a uniform power delay profile, constituted by $1+B \tau_{m}$ multipath components of equal magnitude. 
behaviour of both the transmitter and the receiver of a communication system. Generally speaking, such mappings may be characterized by a large number of parameters corresponding to the various aspects of the mathematical manipulations involved, including channel coding, interleaving, modulation, space-time coding, spreading, as well as the inverse operations invoked in the receiver. From a system design perspective, the choice of any specific transmission scheme $\mathcal{S}$ unambiguously determines the amount of power required by the system in order to operate at the desired performance point $\kappa$, while communicating over a channel $\nu$. More specifically, we may formulate a mathematically tractable correspondence $\gamma(\mathcal{S}, \kappa, \nu)$, where $\gamma$ denotes the minimum signal-to-noise ratio (SNR) required by the transceiver $\mathcal{S}$ in order to support an application $\kappa$, while communicating over a channel $\nu$.

Using the terminology described above, we may now devise a transceiver design criterion in the form of the following optimization problem

$$
\hat{\mathcal{S}}(\kappa, \nu)=\underset{\mathcal{S}}{\operatorname{argmin}} \gamma(\mathcal{S}, \kappa, \nu)
$$

In other worlds, we define the transceiver scheme $\hat{\mathcal{S}}(\kappa, \nu)$, as optimum in the system scenario $\{\kappa, \nu\}$ if $\gamma(\hat{\mathcal{S}}, \kappa, \nu)$ minimizes the required average SNR.

It is important to note that the minimum required SNR $\gamma$ is lowerbounded for any combination of scenarios $\nu$ and $\kappa$. A simple way to verify this is to consider that the minimum SNR required by any constrained system $\{\kappa, \nu\}$ is lower-bounded by the corresponding SNR required to establish an unconstrained communication link of rate $R\left(1-P_{e}\right)$ in an AWGN channel of similar bandwidth Correspondingly, the question arises whether a tight lower bound on the minimum required SNR $\hat{\gamma}(\kappa, \nu)=\min _{\mathcal{S}} \gamma(\mathcal{S}, \kappa, \nu)$ may be readily established. In order to address this problem, in the next section we offer the following important conjecture.

\section{ANALYTICAL CHARACTERIZATION FRAMEWORK}

1) Constrained Capacity Conjecture: Consider the function

$$
\hat{\gamma}(\kappa, \nu)=\min _{\mathcal{S}} \gamma(\mathcal{S}, \kappa, \nu) \text {. }
$$

Specifically, let us keep all but one of the parameters characterizing the system scenario $\{\kappa, \nu\}$ fixed, while sweeping over a range of data-rate values $R$. The resultant function $\hat{\gamma}(r)$, where we define the spectral efficiency $r=R / B$, may be identified as the inverse of the constrained rate-versus-SNR curve reminiscent of the classic Shannon capacity. Evidently, using the Shannon-Hartley theorem, we may postulate that for any single-input single-output system (SISO) scenario $\{\nu, \kappa\}$ we have

$$
\hat{\gamma}(r)>2^{r}-1
$$

Furthermore, we would like to formulate the following proposition.

Proposition 1. Consider the inverse constrained capacity curve $\hat{\gamma}(r)$. For any two points $\gamma_{1}=\hat{\gamma}\left(r_{1}\right)<\gamma_{2}=\hat{\gamma}\left(r_{1}\right)$, we have

$$
\frac{\gamma_{2}}{\gamma_{1}}>\frac{2^{r_{2}}-1}{2^{r_{1}}-1} \text {. }
$$

In other words, for any value of the spectral efficiency $r>0$ the first derivative of the monotonically increasing function $\hat{\gamma}(r)$ is always larger than that exhibited by the inverse of Shannon's AWGN capacity curve $r=\log _{2}(1+\gamma)$.

The inherent validity of Proposition 1 is substantiated by the results obtained in [5]. Although, in this paper we do not provide a formal proof of our conjecture, we would like to offer the following proof outline.

Proof outline. Consider two points $\gamma_{1}=\hat{\gamma}\left(r_{1}\right)<\gamma_{2}=\hat{\gamma}\left(r_{2}\right)$ on a hypothetic inverse constrained capacity curve $\hat{\gamma}(r)$. Let us assume that Proposition 10 does not hold and we have $\gamma_{1}>\gamma_{2}\left(2^{r_{1}}-\right.$ $1) /\left(2^{r_{2}}-1\right)$. From the definition of $\hat{\gamma}(r)$ in (3) we may infer that there exist two transceiver schemes $\mathcal{S}_{1}$ and $\mathcal{S}_{2}$, so that we have
$\gamma_{1}=\gamma\left(\mathcal{S}_{1}, \kappa^{\left(r_{1}\right)}, \nu\right)$ and $\gamma_{1}=\gamma\left(\mathcal{S}_{2}, \kappa^{\left(r_{2}\right)}, \nu\right)$, where $\kappa^{\left(r_{1}\right)}$ and $\kappa^{\left(r_{2}\right)}$ denote two application scenarios, which differ only in terms of the required data-rate $R$ and the corresponding spectral efficiency $r=R / B$. Subsequently, we would like to conjecture that by invoking an additional rate- $\left(r_{1} / r_{2}\right)$ encoding and decoding operation in the architecture of the transceiver scheme $\mathcal{S}_{2}$, we may design a new transceiver scheme $\mathcal{S}_{1}^{\prime}$, which would require the average SNR of

$$
\gamma_{1}^{\prime}=\gamma_{2} \frac{2^{r_{1}}-1}{2^{r_{2}}-1}<\gamma_{1}
$$

in order to support the application scenario $\kappa^{\left(r_{1}\right)}$, which negates the assumption of the point $\left(\gamma_{1}, r_{1}\right)$ belonging to the constrained capacity curve, and thus suggests the validity of Proposition 1

Proposition 2. Let us assume that a point $\gamma_{1}=\hat{\gamma}\left(r_{1}\right)$ lies on the inverse constrained capacity curve $\hat{\gamma}(r)$. Than it follows from Proposition 11 that the curve

$$
\tilde{\gamma}(r)=\gamma_{1} \frac{2^{r}-1}{2^{r_{1}}-1}
$$

constitutes the lower bound of the SNR value required to achieve a rate of $r>r_{1}$, as well as the upper bound of the SNR values required to achieve a rate of $r<r_{1}$.

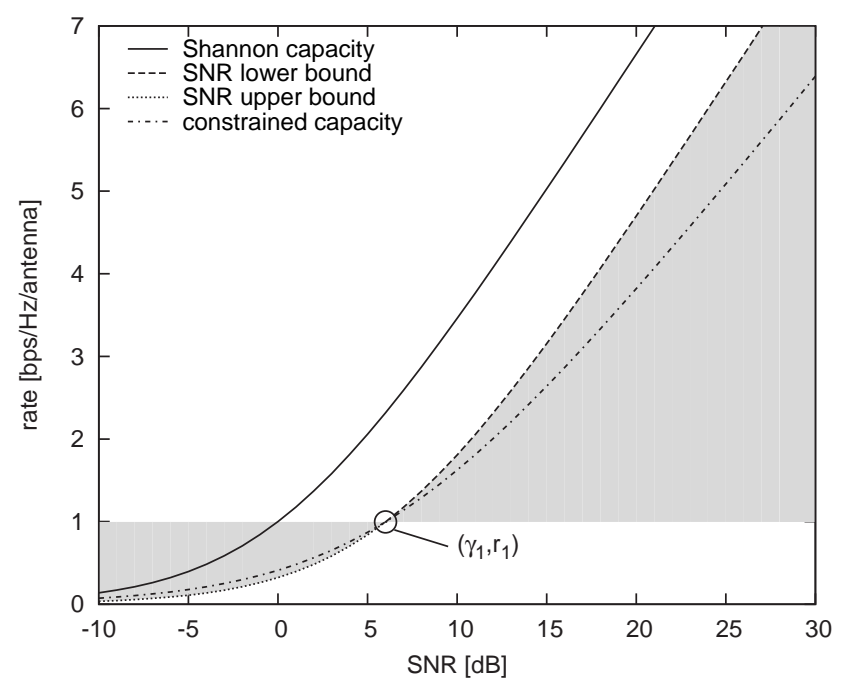

Fig. 2. Constrained capacity bounds.

Proposition 2 is illustrated in Figure 2 where the known constrained capacity point $\left(\gamma_{1}, r_{1}\right)$ determines the bound $\tilde{\gamma}(r)$ on the average SNR, which is required in order to support a spectral efficiency of $r$. Correspondingly, the shaded areas in Figure 2 correspond to the feasibility regions, where the inverse constrained capacity curve $\hat{\gamma}(r)$ may be situated.

2) Outage probability-based error rate: Consider a rate$\left(1 / r_{d}\right)$ transceiver scheme $\mathcal{S}$ communicating in an uncorrelated Rayleigh channel using bursts of $r_{d}$ BPSK symbols. Specifically, in each burst a single bit of information is encoded using a rate- $\left(1 / r_{d}\right)$ repetition code into a length- $r_{d}$ BPSK signal vector. We assume the channel to be perfectly known at the receiver. Correspondingly, a single transmitted bit of information $s$ is decoded at the receiver using an Optimum Ratio Combining (ORC) method, which yields

$$
\hat{s}=\frac{\sum_{k=1}^{r_{d}} h_{k}^{*} s_{k}^{(r)}}{\sum_{k=1}^{r_{d}}\left|h_{k}\right|^{2}+N_{0}},
$$

where $s_{k}^{(r)}=h_{k}\left(\mathcal{S}^{(t)}(s)\right)_{k}+w_{k}$ denotes the $k$ th element of the signal vector recorded at the receiver, $h_{k}$ denotes the $k$ th element 
of the Rayleigh i.i.d. vector of channel coefficients, while $N_{0}$ is the AWGN variance.

The error probability $P_{e}=P(\hat{s} \neq s)$ in this case is determined by the outage probability [6], which may be approximated as

$$
P_{e} \approx\left(\frac{1}{1+r_{d} \gamma}\right)^{r_{d}}\left(\begin{array}{c}
2 r_{d}-1 \\
r_{d}
\end{array}\right)
$$

Inverting (9) yields the minimum average SNR that has to be satisfied in order to maintain the error probability of $P_{e}$. Namely, we have

$$
\gamma=\frac{1}{r_{d}}\left[\left(\begin{array}{c}
2 r_{d}-1 \\
r_{d}
\end{array}\right)^{1 / r_{d}} P_{e}^{-1 / r_{d}}-1\right] .
$$

Importantly, the outage probability associated with a fading channel exhibiting a diversity order of $r_{d}$ constitutes a lower bound on the error rate attainable by any transceiver scheme. We may therefore utilize (10) in conjunction with Proposition 2 in order to obtain a bound on the minimum required SNR for a wide range of system scenarios $\{\kappa, \nu\}$. In this context, it is plausible that the key challenge in deriving the desired bounds is constituted by the correct estimation of the diversity order, which may be attained in any system scenario $\{\kappa, \nu\}$.

3) Signal dimensionality: The dimensionality and the mathematical properties of the binary vectors $s$ and $\hat{s}$ of Equation (1), as well as the intermediate transmitted and received signals $s^{(t)}=$ $\mathcal{S}^{(t)}(s)$ and $\boldsymbol{s}^{(r)}=\boldsymbol{H} \mathcal{S}^{(t)}(\boldsymbol{s})$ are generally determined by the characteristics of the transceiver scheme $\mathcal{S}$ and the channel matrix $\boldsymbol{H}$. In the context of wireless communication systems we define the spectral bandwidth $B \gg \frac{1}{T}$ occupied by the radio frequency signal as the width (in Hertz) of the frequency interval, where most of the desired signal's energy is concentrated ${ }^{2}$. Using the classic Nyquist theorem [6], we may unambiguously describe the band-limited continuous RF signals by their discrete complex-valued baseband equivalents.

Correspondingly, the resultant discrete transmitted and received signals may be formulated as $D_{F}^{(t)} / 2$ - as well as $D_{F}^{(r)} / 2$-element complex-valued column vectors $s^{(t)}$ and $s^{(r)}$, respectively. In a simple SISO non-dispersive scenario, we have $D_{F}^{(t)}=D_{F}^{(r)}=2 B T$. Generally speaking, however, the signal dimensions $D_{F}^{(t)}$ and $D_{F}^{(r)}$ may be quite different from each other, depending on the amount of time- and frequency-domain spreading, as well as the on spatial dimensionality of the system considered. Specifically, in the case of a $\left(n_{t} \times n_{r}\right)$-dimensional MIMO multipath Rayleigh-fading channel $\nu=$ $\left\{f_{d}, \tau_{m}\right\}$ we have $D_{F}^{(t)}=2 n_{t} B T$ and $D_{F}^{(r)}=2 n_{r}\left(B+f_{d}\right)\left(T+\tau_{m}\right)$.

4) Spreading and coding: Consider the transmitter $\mathcal{S}^{(t)}$ of Equation (1), which invokes a channel coding of rate $\nu_{c}$ followed by the modulation operation, which maps $\nu_{m}$ bits onto a single complex-valued symbol as well as by a spreading operation having a spreading factor of $N_{s}$. Furthermore, we may consider a $n_{t}$ antenna-aided transmitter invoking a space-time code of rate $\nu_{s}$. The resultant transmitter scheme $\mathcal{S}^{(t)}$ may be described by a mapping between the $\left(k=R T=\nu_{c} \nu_{m} \nu_{s} n_{t} B T / N_{s}\right)$-element binary vector $s$ and the $\left(n_{t} B T\right)$-element complex-valued transmitted signal $\boldsymbol{s}^{(t)}$ of Equation (1). We may therefore conclude that the transmitter $\mathcal{S}^{(t)}$ exhibits a transmission rate of $R=\nu_{c} \nu_{m} \nu_{s} n_{t} B / N_{s}$ bits per second.

Furthermore, taking into account the dimensionality of the channel matrix $\boldsymbol{H}$ in Equation (1), we may formulate the corresponding transformation as $\boldsymbol{s}^{(r)}=\boldsymbol{H} \mathcal{S}^{(t)}(s)$, which constitutes a mapping between the $\left(\nu_{c} \nu_{m} \nu_{s} n_{t} B T / N_{s}\right)$-element binary vector $s$ and the $\left[n_{r}\left(B+f_{D}\right)\left(T+\tau_{m}\right)\right]$-element complex-valued received signal vector $s^{(r)}$. Once again, invoking the methodology proposed in [7] we may formulate the following conjecture

Proposition 3. The transformation $\boldsymbol{s}^{(r)}=\boldsymbol{H} \mathcal{S}^{(t)}(\boldsymbol{s})$ may be interpreted as a transceiver scheme characterized by an overall

\footnotetext{
${ }^{2}$ The basic assumption of having $W \gg \frac{1}{T} \Rightarrow \frac{1}{W} \ll T$ has to be
} maintained in order to avoid interference between subsequent time-slots. coding rate of

$$
\tilde{\nu}_{c}=\frac{R T}{\nu_{m} n_{t} B T},
$$

as well as an overall spreading factor of

$$
\tilde{N}_{s}=N_{s} \frac{n_{r}\left(B+f_{d}\right)\left(T+\tau_{m}\right)}{B T} .
$$

5) Maximum free distance: An important characteristic of the coding component in the transceiver scheme $\mathcal{S}^{(t)}$ of Equation (1) is constituted by the maximum free distance $d$ that is achievable by a code of length $n=n_{t} \nu_{m} B T$ and rate $\nu_{c}$ [8]. An approximate solution to the problem of calculating the maximum free distance $d$ may be found in [9], while the best known theoretical upper bound is derived in [10]. Importantly however, in most practical scenarios the effectively achievable maximum free distance is constrained by the affordable decoding complexity $\Omega$. Based on the properties of Low Density Parity Check (LDPC) codes [11] as well as on the empirical evidence, we would like to formulate the following conjecture

Proposition 4. The realistically realizable free distance of a code is upper bounded by the logarithm of the decoder complexity per bit. Specifically, we have

$$
\tilde{d}(n, r)=\min \left[\log \Omega, d\left(n, \nu_{c}\right)\right],
$$

where $\Omega$ denotes the maximum number of binary operations per decoded bit performed by the decoder, while $d\left(n, \nu_{c}\right)$ is the approximate maximum free distance calculated using the method of [9].

6) Diversity: The diversity order is a property of the channel matrix $\boldsymbol{H}$ of Equation (1), which characterizes the statistical distribution of its output energy. Specifically, the stochastic channel matrix $\boldsymbol{H}=\left\{h_{i j}\right\}$ exhibits the diversity order of $r_{d}$, if $\sum_{i} \sum_{j}\left|h_{i j}\right|^{2}$ constitutes a $\chi_{2 r_{d}}^{2}$-distributed random variable. Alternatively, we may define the channel's diversity order as $r_{d}=\operatorname{rank}\left(E\left\{\boldsymbol{H} \boldsymbol{H}^{*}\right\}\right)$, where $\boldsymbol{H}^{*}$ is the adjoint of the matrix $\boldsymbol{H}$, while $E\{\cdot\}$ denotes the ergodic expectation.

Generally speaking, the diversity stems from the stochastic variability of the channel coefficient across time, frequency and spatial dimensions. For instance, the frequency diversity is determined by the number of resolvable multipath components in the channel's power delay profile [6]. Correspondingly, assuming a rich scattering propagation environment [12], the frequency diversity order pertaining to a channel of bandwidth $B$ and exhibiting a delay spread of $\tau_{m}$ may be expressed as $r_{d}^{(f)}=1+B \tau_{m}$ [6]. Likewise, the time diversity order is determined by the number of resolvable samples in the temporal fading process' power spectrum density, and may be expressed as $r_{d}^{(t)}=1+f_{d} T$. Finally, the spatial diversity order is determined by the number of uncorrelated spatial links between the transmit and receive antennas, and may be expressed as $r_{d}^{(s)}=\alpha n_{t} n_{r}$, where $\alpha$ denotes the spatial correlation coefficient [13]. Correspondingly, in the case of a $\left(n_{t} \times n_{r}\right)$ Rayleigh-fading MIMO channel $\nu=\left\{f_{d}, \tau_{m}\right\}$, the total diversity order may be expressed as the product of space, time and frequency diversity orders, which yields

$$
r_{d}=r_{d}^{(t)} r_{d}^{(f)} r_{d}^{(s)}=\alpha n_{t} n_{r}\left(1+T f_{d}\right)\left(1+W \tau_{m}\right)
$$

Importantly, the practically achievable diversity order is typically lower. In the first stage of the detection process, the received signal may benefit from power combining, resulting in a combining diversity order, which may be expressed as $r_{d}^{(c)} \leq \min \left(N, r_{d}\right)$, where $N$ is the overall spreading factor, while $r_{d}$ is the total diversity order defined in (14. Subsequently, in the second stage a further increase in the attainable diversity order may be achieved by exchange of soft likelihood information between encoded bits associated with independently faded received symbols [14]. The maximum degree of diversity, which may be attained in the decoding process is denoted as the decoding diversity order, which may be expressed as $r_{d}^{(d)} \leq \min \left(\tilde{d}, r_{d}\right)$. Ultimately, we offer the following conjecture 
Proposition 5. The maximum achievable diversity order of the system may be expressed as the product of the combining and decoding diversity orders, which yields

$$
\tilde{r}_{d}=\min \left(r_{d}, \tilde{d} \cdot \tilde{N}_{s}\right),
$$

where $r_{d}$ is the total diversity order defined in Equation (14), $\tilde{d}$ is the complexity-constrained maximum free distance of Equation (13), while $N_{s}$ denotes the total cumulative spreading factor of Equation (12).

\section{CONSTRAINED CAPACITY}

Let us now consider the system scenario of

$$
\{\kappa ; \nu\}=\left\{T, B, R, P_{e}, \Omega, n_{t}, n_{r} ; \alpha, f_{D}, \tau_{m}\right\} .
$$

Our objective is to calculate the lower bound on the minimum SNR required to support the system scenario $\{\kappa, \nu\}$ of (16). Specifically, from (11) and (12) we have $k=R T, n=\nu_{m} n_{t} T B$, as well as $\tilde{\nu}_{c}=k / n$ and $\tilde{N}_{s}=n_{r}\left(B+f_{D}\right)\left(T+\tau_{m}\right) / B T$, where we assume $N_{s}=1$.

The corresponding effective maximum free distance $\tilde{d}$ may be calculated using (13), while the maximum achievable diversity order may be calculated as in (15), yilding

$$
\tilde{r}_{d}=\min \left(\alpha n_{t} n_{r}\left(1+T f_{d}\right)\left(1+\tau_{m} B\right), \tilde{d} \cdot \tilde{N}_{s}\right) .
$$

Subsequently, the minimum SNR required by a rate- $1 / r_{d}$ reference system of Section III-2 may be expressed as in 10, yielding

$$
\tilde{\gamma}=\frac{1}{r_{d}}\left[\left(\begin{array}{c}
2 r_{d}-1 \\
r_{d}
\end{array}\right)^{1 / r_{d}} P_{e}^{-1 / r_{d}}-1\right] .
$$

Finally, we invoke Proposition 2 in order to calculate the minimum SNR required to operate in the system scenario $\{\kappa, \nu\}$ of (16), hence, we have

$$
\gamma(r)=\frac{\tilde{\gamma} \sqrt{n_{r}}}{\tilde{N}_{s}} \frac{2^{\tilde{\nu}_{c}}-1}{2^{1 / r_{d}}-1}
$$

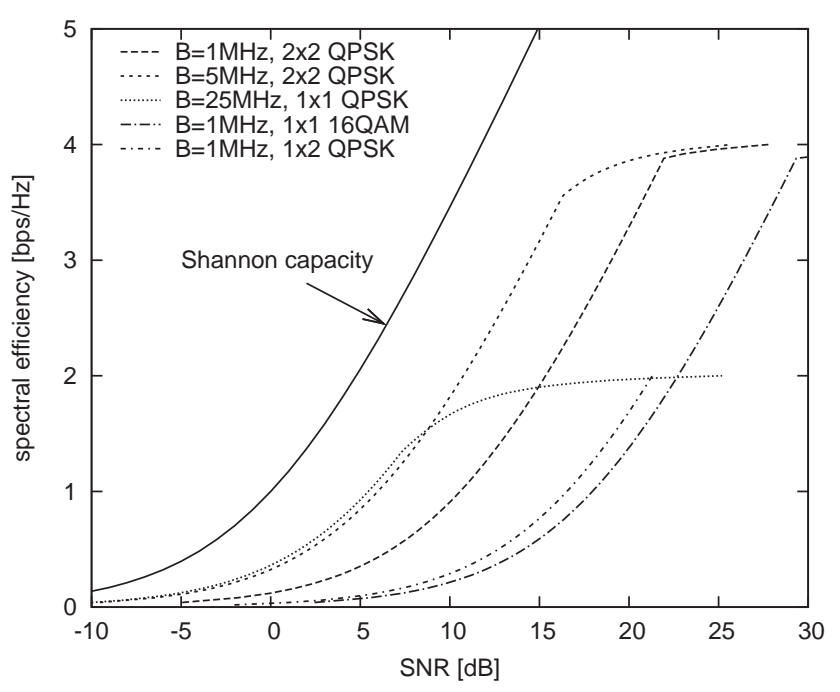

Fig. 3. Constrained capacity of wireless communication systems assuming the common parameters: $n=100, \Omega=1000, \alpha=1, f_{D}=10 \mathrm{~Hz}, \tau_{m}=6 \mu \mathrm{sec}$, as well as the scenario-specific parameters summarized in Table [

1) Example: Let us now invoke the above methodology for the sake of characterizing a host of realistic system scenarios. The results of Figure 3 were computed by substituting the system scenario parameters of (16) into Equations (17)-(19). Specifically, each curve on Figure 3 was obtained by sweeping the number of information
TABLE I

TEST-CASE SYSTEM SCENARIOS PARAMETERS

\begin{tabular}{c|cccc} 
& $\mathrm{B}[\mathrm{MHz}]$ & $n_{t}$ & $n_{r}$ & $\nu_{m}$ \\
\hline 1 & 1 & 2 & 2 & 2 \\
2 & 5 & 2 & 2 & 2 \\
3 & 25 & 1 & 1 & 2 \\
4 & 1 & 1 & 1 & 4 \\
5 & 1 & 1 & 2 & 1
\end{tabular}

bits $k$ between the values of 1 and $n=\nu_{m} n_{t} B T$ and subsequently calculating the minimum SNR required to support the resultant system scenario $\{\kappa, \nu\}$, exhibiting a data-rate of $R=k / T$ bps and a spectral efficiency of $k / B T \mathrm{bps} / \mathrm{Hz}$. All test-case scenarios assumed the following common parameters: $n=100, \Omega=1000, \alpha=1, f_{D}=$ $10 \mathrm{~Hz}, \tau_{m}=6 \mu \mathrm{sec}$, as well as the scenario-specific parameters summarized in Table \

Observe, that the particular choice of the various system parameters, such as the channel bandwidth $B$ may have a dramatic effect on the system's constrained capacity. The proposed methodology, may therefore, be employed for the design of locally-optimum transceiver schemes in the context of multifunctional heterogeneous wireless networks.

2) Conclusions and Future Work: We have proposed an analytical framework for the characterization of wireless transceivers. The proposed methodology may be readily utilized for the design and optimization of wireless communication systems across a wide range of application and channel scenarios.

Importantly, this manuscript provides a proof of concept, while relying on a number of important conjectures, which require a more detailed theoretical, as well as empirical justification. The thorough analysis of the aforementioned propositions will form part of our future work.

\section{REFERENCES}

[1] G. Gow and R. Smith, Mobile and Wireless Communications: An Introduction. Open University Press/McGraw-Hill, 2005.

[2] J. Andrews, S. Shakkottai, R. Heath, N. Jindal, M. Haenggi, R. Berry, D. Guo, M. Neely, S. Weber, S. Jafar, and A. Yener, "Rethinking information theory for mobile ad hoc networks," IEEE Communications Magazine, vol. 46, no. 12, pp. 94-101, December 2008.

[3] S. McCann, W. Groting, A. Pandolfi, and E. Hepworth, "Next generation multimode terminals," in IEE International Conference on $3 G$ Mobile Communication Technologies, London, UK, 2004, pp. 143-147.

[4] B. Fette, Cognitive Radio Technology. Elsevier Science and Technology Books, 2006.

[5] J. Akhtman and L. Hanzo, "Closed-form approximation of MIMO capacity," Electronics Letters, vol. 45, no. 1, pp. 68-69, 12009.

[6] J. Proakis, Digital Communications. McGraw-Hill, 2001.

[7] V. V. Veeravalli and A. Mantravadi, "The coding-spreading tradeoff in CDMA systems," IEEE Journal on Selected Areas in Communications, vol. 20, no. 2, pp. 396-408, Feb. 2002.

[8] T. Moon, Error Correction Coding: Mathematical Methods and Algorithms. Wiley-Interscience, 2006.

[9] J. Akhtman, N. Bonello, R. G. Maunder, and L. Hanzo, "Closed-form approximation of maximum free distance for binary block codes," in VTC'09 Fall, Anchorage, Alaska, USA, 20-23 September 2009.

[10] R. McEliece, E. Rodemich, H. Rumsey, and L. Welch, "New upper bounds on the rate of a code via the Delsarte-MacWilliams inequalities," IEEE Transactions on Information Theory, vol. 23, no. 2, pp. 157-166, March 1977.

[11] N. Bonello, S. Chen, and L. Hanzo, "On the design of low-density paritycheck codes," IEEE Communications Magazine, 2009, submitted.

[12] A. Algans, K. Pedersen, and P. Mogensen, "Experimental analysis of the joint statistical properties of azimuth spread, delay spread, and shadow fading," IEEE Journal on Selected Areas in Communications, vol. 20, no. 3, pp. 523-531, April 2002.

[13] W. Weichselberger, W. Herdin, H. Ozcelik, and E. Bonek, "A stochastic MIMO channel model with joint correlation of both link ends," IEEE Transactions on Wireless Communications, vol. 5, no. 1, pp. 90-100, Jan. 2006.

[14] E. Biglieri, J. Proakis, and S. Shamai, "Fading channels: informationtheoretic and communications aspects," IEEE Transactions on Information Theory, vol. 44, no. 6, pp. 2619-2692, Oct. 1998. 